\title{
Integrated Transcriptome-Wide Profiling And Protein Structure Analysis of Pathogenic Genes In Venous Thromboembolism
}

\author{
Jing Ding \\ Shanghai Institue for Biomedical and Pharmaceutical Technologies \\ Yan Zhang \\ Shanghai Institue for Biomedical and Pharmaceutical Technologies \\ Yan Che ( $\nabla$ cheyan2004@163.com) \\ Shanghai Institue for Biomedical and Pharmaceutical Technologies
}

\section{Research}

Keywords: Venous thromboembolism, Transcriptome-wide profiling, Whole exome sequencing, Protein structure, HNMT

Posted Date: October 25th, 2021

DOI: https://doi.org/10.21203/rs.3.rs-709943/v2

License: () (i) This work is licensed under a Creative Commons Attribution 4.0 International License. Read Full License 


\section{Abstract}

Background: Genetic factors are considered to determine the balance of the coagulation and anticoagulation processes, yet the genetic variants related to venous thromboembolism (VTE) remain unclear. This study aimed to investigate the potential molecular mechanisms and pathogenic mutations associated with VTE by determining VTE-related differentially expressed genes (DEGs) by transcriptome-wide profiling and assaying protein structure in VTE.

Methods: Two gene expression datasets, GSE48000 and GSE19151, were accessed from the Gene Expression Omnibus (GEO) database to obtain gene expression data associated with VTE. We identified the DEGs between VTE patients and healthy people using R and performed functional enrichment analysis, including Gene Ontology (GO) and Kyoto Encyclopedia of Genes and Genomes (KEGG) analysis. Then, whole-exome sequencing (WES) was performed for 25 VTE patients and 17 normal cases, and the structural locations of pathogenic missense mutations were identified using pyMOL. Finally, DGIdb database was used to select candidate drugs for the treatment of VTE.

Results: A total of 232 DEGs were identified from the GEO database. The significant function of these DEGs was mostly involved in RNA catabolic process and ribosome pathway. Notably, the results of WES for DEGs and protein structure analysis showed that Histamine N-Methyltransferase (HNMT) (chr2: 138759649 C>T, rs11558538) may be a main predisposing factor for VTE. In addition, Amodiaquine, Harmaline, Aspirin, Metoprine, Dabigatran, and Diphenhydramine were screened for VTE therapy.

Conclusion: The results showed that HNMT (chr2: 138759649 C>T, rs11558538) may be potential target for the diagnosis and treatment of VTE.

\section{Introduction}

Venous thromboembolism (VTE) is the third most common cardiovascular disease worldwide, which manifests as deep-vein thrombosis, pulmonary embolism, or both[1, 2]. Various epidemiological studies have demonstrated that the incidence of VTE is characterized by a remarkable number of genetic and environmental factors. In early epidemiological studies, the highest incidence of VTE was in Africa, followed by Caucasians and was lower for Asian[3]. With increased awareness of the diagnosis and management of VTE, the incidence in Asian has increased in recent years[4].

While VTE is classified as a complex, multifactorial and polygenic disease, common mechanisms driven VTA have been confirmed, such as gene-gene and gene-environment interactions[5]. Stasis, vessel damage, and a hypercoagulable state are three widely accepted mechanisms related to the occurrence of VTE[6]. Genetic epidemiological studies have revealed that genetic conditions are significant risk factors for VTE, accounting for up to $50 \%$ of all VTE patients including anticoagulant protein deficiency trapping (deficiency of protein C, protein S, and antithrombin), factor V Leiden mutation (FVL) (c.1601G > A, p.R534Q), prothrombin G20210A mutation (FII G20210A), hyperhomocysteinemia, elevated coagulation factors VIII, IX, X, histidine rich glycoprotein, and ABO blood group [7, 8]. However, only a few genetic factors have been considered and the distribution of FVL and FII G20210A mutation depends on ethnic group, race, or geographical region, suggesting that there is still an urgent need to identify VTE pathogenic genetic factors[9].

With the development of gene chip technology, large-scale deep sequencing and bioinformatics analysis, scientists now have rich datasets for answering biological questions, including information on DEGs, pathways, and even targeted drugs associated with disease development[10]. Whole-exome sequencing and protein structure analysis can detect potentially important mutations that have not been reported, which is, hence, of vital significance for VTE patients to enrich anticoagulation and the use of catheter-directed thrombolytic therapy[11].

In this study, we used the GSE48000 and GSE19151 datasets downloaded from the Gene Expression Omnibus to identify DEGs associated with VTE. GO and KEGG enrichment analyses of these DEGs were then performed. WES for a total of 25 VTE patients and 17 normal people was performed to screen out pathogenic mutations in VTE associated DEGs. Based on our findings, HNMT(chr2: 138759649 C > T, rs11558538) appeared to be a genetic susceptibility risk factor for VTE, representing a novel, potentially druggable target for the treatment of VTE.

\section{Materials And Methods}

\subsection{Patients samples}

Twenty-five VTE and 17 normal patients admitted to participating hospitals from July 2015 to December 2018 were selected as study subjects. All patients were confirmed for VTE by B-ultrasound scan or CT examination, and basic characteristics of the recruited VTE patients were recorded, including gender, age, medication history, disease history, history of cardiovascular diseases, and history of chronic obstructive pulmonary disease. All patients signed informed consent documents, and this study was approved by the ethics committee of the Shanghai Institute of Planned Parenthood Research and ethical committees of participating hospitals.

\subsection{Extraction of datasets}

Gene expression profiles were mined from the GEO (GEO: www.ncbi.nlm.nih.gov/geo/) database, which distributes high-throughput gene expression and other functional genomics datasets, using the following keywords: 'VTE' and 'Homo sapiens' [12]. Two datasets, GSE48000 and GSE19151, were identified for this study. The gene expression profile dataset of GSE48000 included 40 high-risk VTE cases and 25 healthy controls and had data on whole blood-derived RNA samples sequenced using a GPL10558 Illumina Human HT-12 V4.0 expression bead chip[13]. The GSE19151 dataset was generated on the GPL571[HGU133A_2] Affymetrix Human Genome U133A 2.0 Array platform. This dataset contained 70 adults with VTE cases and 63 healthy controls[14]. 
The original raw expression data at the probe level was downloaded as CEL files and pre-processed and normalized with RMA using the 'affy' package in R version 4.0.2, followed by converting the data into corresponding gene expression data based on the different platform specifications[15]. The Pearson's correlation coefficient was determined to validate the intra-group data repeatability and heatmap generation was visualized based on the 'heatmap' $\mathrm{R}$ package[16]. Principal component analysis (PCA) was conducted to view the clustering trends according to sample-to-sample distances using the 'ggord' package in $\mathrm{R}[17]$.

\subsection{Identification of DEGs}

The 'limma' package in R program was applied to screen DEGs between VTE samples and normal samples[18]. A two-tailed t-test was performed to examine DEGs by $\log 2$ (Fold Change) $>1$ or $<-1$ and adjusted $P$ value $<0.05$. Genes satisfying these conditions were grouped separately as $D E G s$ by volcano plot in $R$ [19].

\subsection{Gene ontology and KEGG pathway enrichment analysis}

$\mathrm{GO}$ is used to describe the biological processes (BPs), molecular functions (MFs), and cellular components (CCs) of gene products in a hierarchical ontology[20]. Signaling pathway analysis was conducted to map DEGS to the Kyoto Encyclopedia of Gene and Genomes (KEGG), which is a pathway-related database for systematic and comprehensive analysis of gene functions[21]. GO and KEGG pathway enrichment analyses were performed using the 'cluster Profiler' package in $\mathrm{R}$ version 4.0.2 and $\mathrm{P}$ values less than 0.05 were considered statistically significant[22]. A GO network was visualized using the Metascape database (http://metascape.org) to validate our results[23].

\subsection{Whole-exome sequencing for DEGs}

DNA was extracted from each patient using a DNA extraction kit (Qiagen, Hilden, Germany) from whole blood. Library construction, WES, and data analysis were conducted by iGeneTech in Shanghai. Then, $200 \mathrm{ng}$ of genomic DNA from each individual was sheared by Biorupter (Diagenode, Belgium) to acquire 150-200 bp fragments. The ends of DNA fragment were repaired and Illumina adapters were added (Fast Library Prep Kit, iGeneTech, Beijing, China). After sequencing libraries were constructed, whole exomes were captured using the AlExome Enrichment Kit V1 (iGeneTech, Beijing, China) and libraries sequenced on an Illumina NovaSeq 6000 (Illumina, San Diego, CA) Next-Generation sequencing platform in the 150 bp PE mode. Bioinformatics analysis was performed to analyze nonsynonymous mutations including single-nucleotide polymorphisms (SNPs) and insertion-deletions (INDELs) using GATK (Genome Analysis Toolkit). All allele frequency data for DEG mutations were compared with the 1000 Genomes Project (http://www.1000genomes.org) and Exome Aggregation Consortium ExAC (http://exac.broadinstitute.org).

\subsection{Protein Structure Modeling and Molecular Docking}

The three-dimensional structure diagram of HNMT was generated using swiss model (http://www.swissmodel.expasy.org) and pymol v2.4(https://pymol.org/2/). The DGldb database (https://dgidb.org/) was used to select drugs based on the genes that served as promising targets[24].

\subsection{Molecular Docking}

Ligand docking of HNMT and drugs was performed with default parameters using AutoDock molecular docking software (version 4.2) and the coordinates and box size were finalized according to ligand location[25].

\subsection{Statistical Analysis}

DEGs were selected based on t test. The whole genome GO categories and pathogenic mutations for these DEGs were identified using Fisher's exact test. The significance level for statistical tests was set at less than $0.05(P<0.05)$.

\section{Results}

\subsection{Pearson's Correlation Testing and PCA}

Pearson's correlation test showed strong correlations between VTE and control samples in the GSE48000 dataset (Fig. 1A). The PCA profile for the GSE48000 data revealed that the distances between samples were small in the VTE groups and control groups, respectively (Fig. 1B). Pearson's correlation analysis also indicated strong correlations for the GSE19151 data among the samples in the VTE group and control group, respectively (Fig. 1C). The close distance in the dimension of PCA illustrated the acceptable data repeatability between samples in the VTE group and control group for the GSE19151 dataset (Fig. 1D).

\subsection{Identification of DEGs in VTE}

As shown in Fig. 2, a total of 232 genes were designated as DEGs in the VTE group when compared with the control group. The volcano plots in this figure present the DEGs with a cutoff criteria of having an adjusted P-value $<0.05$ and $\log _{2}$ fold changel $>1$ in the GSE48000 and GSE19151 datasets (Fig. $2 A$ and 2B). As examples of these differences, the top 10 differentially expressed genes are reported in Table 1. 
Table 1

Top 10 most upregulated DEGs and top 10 most downregulated DEGs in

VTE

\begin{tabular}{|llll|}
\hline Gene name & Log2 fold change & Adjusted P-value & Gene Expression \\
\hline EVI2A & 2.15595960 & $2.46 \times 10^{-13}$ & Upregulation \\
\hline RPL9 & 1.89195402 & $1.48 \times 10^{-18}$ & Upregulation \\
\hline IFI27 & 1.87618320 & $1.88 \times 10^{-5}$ & Upregulation \\
\hline RPL31 & 1.77436415 & $3.44 \times 10^{-14}$ & Upregulation \\
\hline NDUFA4 & 1.74588189 & $7.28 \times 10^{-17}$ & Upregulation \\
\hline IGFBP1 & 1.74512022 & $1.33 \times 10^{-7}$ & Upregulation \\
SNORD8 & 1.71070142 & $5.02 \times 10^{-5}$ & Upregulation \\
RPS7 & 1.68354151 & $2.35 \times 10^{-13}$ & Upregulation \\
XK & 1.63316845 & $1.00 \times 10^{-11}$ & Upregulation \\
RPS15A & 1.51993276 & $1.20 \times 10^{-15}$ & Upregulation \\
FOS & -1.37065523 & $1.43 \times 10^{-7}$ & Downregulation \\
JMJD1C & -1.30709903 & $3.65 \times 10^{-6}$ & Downregulation \\
\hline TMEM259 & -1.05424088 & $5.98 \times 10^{-24}$ & Downregulation \\
\hline DCK & -1.04088634 & $4.66 \times 10^{-13}$ & Downregulation \\
CD46 & -1.16530934 & $7.51 \times 10^{-6}$ & Downregulation \\
SNX10 & -1.14759082 & $2.10 \times 10^{-5}$ & Downregulation \\
UBXN4 & -1.14648762 & $1.22 \times 10^{-5}$ & Downregulation \\
LSP1 & -1.13523701 & $3.46 \times 10^{-9}$ & Downregulation \\
\hline
\end{tabular}

\subsection{Enrichment of DEGs by GO and KEGG Analysis}

Gene functional enrichment analysis was performed to analyze the biological connections of these DEGs. Results of Gene Ontology (GO) enrichment analysis revealed that RNA catabolic process, viral gene expression, SRP-dependent cotranslational protein targeting to membrane, and viral transcription were the main biological processes (BPs) (Fig. 3A), and structural constituent of ribosome, cytochrome-c oxidase activity, and heme-copper terminal oxidase activity were the most enriched categories of molecular functions for these DEGs (Fig. 3B). The variations in cell component (CC) of DEGs were enriched largely in ribosome and hemoglobin complex (Fig. 3C). KEGG pathway analysis indicated that these DEGs were mainly involved in particular pathways, such as the ribosome, Huntington disease, and oxidative phosphorylation (Fig. 3D). Metascape was used to visualize these gene enrichment analyses to verify our results from R (Fig. 3E). We found that these DEGs were enriched in amino acid deficiency, ribosomal complex, oxidative phosphorylation, rRNA transcript, and blood coagulation.

\subsection{Identification of Probable Disease-causing DEGs by WES}

WES revealed 48 mutations of DEGs in the VTE group. The mutation types and the $\log _{2}$ fold change are shown in Table 2 . Because nonsynonymous mutations are most likely to affect protein function, we focused on the four SNP variants corresponding with four DEGs in the VTE group. These were HNMT (ch2: $138759649 \mathrm{C}>\mathrm{T}, \mathrm{rs} 11558538$, adjusted P-value $\left.=1.2 \times 10^{-9}\right)$, POLL (chr10: $103340056 \mathrm{G}>\mathrm{A}$, rs3730477, adjusted P-value $\left.=5.12 \times 10^{-4}\right), \mathrm{ZNF} 292($ chr6: $87925827 \mathrm{~A}>\mathrm{G}, \mathrm{rs} 9362415$, adjusted P-value $\left.=2.95 \times 10^{-8}\right)$, and DPCD (chr10: $103361088 \mathrm{C}>\mathrm{T}$, rs7874, adjusted P-value $\left.=4.36 \times 10^{-5}\right)$. The adjusted Pvalue of HNMT was the lowest in this study. Functional analysis showed that most disease-causing DEGs were involved in anemia, sickle cell, pulmonary thromboembolisms, heparin-induced thrombocytopenia, thrombophilia, and so on, as shown by Metascape functional analysis (Fig. 4). As summarized in Table 3, we found that HNMT was expressed in heparin-induced thrombocytopenia, dermatitis, and atopic cases, conditions that may have strong impacts on VTE. 
Table 2

Probable disease-causing DEGs of VTE

\begin{tabular}{|c|c|c|c|c|c|c|c|c|c|c|}
\hline Gene & Chr & SNP & Mut_type & Mutation & Location & Position & Func.ref & Exonicfunc.ref & $1 \mathrm{KG}$ & ExAC_Al \\
\hline HNMT & $\operatorname{ch} 2$ & rs11558538 & SNP & $\mathrm{C} / \mathrm{T}$ & ontarget & 138759649 & exonic & $\begin{array}{l}\text { nonsynonymous } \\
\text { SNV }\end{array}$ & 0.0595048 & 0.1017 \\
\hline \multirow[t]{2}{*}{ USP14 } & chr18 & rs56806027 & SNP & $\mathrm{T} / \mathrm{A}$ & flank150 & 204815 & intronic & & 0.68111 & \\
\hline & & rs57035428 & InDel & T/TAAAAA & flank150 & 204816 & intronic & & & \\
\hline SERPING1 & chr18 & rs17072114 & SNP & $\mathrm{T} / \mathrm{C}$ & flank150 & 61584817 & intronic & & 0.171326 & \\
\hline \multirow[t]{6}{*}{ UBXN4 } & chr2 & rs80198954 & SNP & $\mathrm{C} / \mathrm{A}$ & ontarget & 136511842 & exonic & $\begin{array}{l}\text { synonymous } \\
\text { SNV }\end{array}$ & 0.0373403 & 0.019 \\
\hline & & rs74265494 & SNP & $G / A$ & ontarget & 136511886 & intronic & & 0.0397364 & 0.0191 \\
\hline & & rs372143998 & InDel & $\mathrm{GT} / \mathrm{G}$ & flank150 & 136527319 & intronic & & & 0.2468 \\
\hline & & rs200613240 & InDel & $\mathrm{T} / \mathrm{TA}$ & flank150 & 136529897 & intronic & & & \\
\hline & & rs78878675 & SNP & $\mathrm{G} / \mathrm{A}$ & ontarget & 136530157 & intronic & & 0.0397364 & 0.019 \\
\hline & & rs78339162 & SNP & $A / G$ & flank150 & 136533993 & intronic & & 0.0397364 & \\
\hline \multirow[t]{2}{*}{ ZNF271P } & chr18 & rs12965288 & SNP & $\mathrm{C} / \mathrm{A}$ & ontarget & 32888090 & ncRNA_exonic & & 0.249401 & \\
\hline & & rs34841246 & SNP & $\mathrm{C} / \mathrm{A}$ & ontarget & 32888546 & ncRNA_exonic & & 0.220847 & \\
\hline SELP & chr1 & rs35706397 & InDel & $\mathrm{T} / \mathrm{TA}$ & ontarget & 169560727 & intronic & & 0.268371 & 0.3319 \\
\hline \multirow[t]{2}{*}{ GYPA } & chr4 & rs62334651 & SNP & $\mathrm{T} / \mathrm{C}$ & flank150 & 145040784 & intronic & & 0.402556 & \\
\hline & & rs62334653 & SNP & $G / A$ & flank151 & 145041036 & intronic & & & \\
\hline \multirow[t]{2}{*}{ TMEM259 } & chr19 & rs2240161 & SNP & $A / G$ & ontarget & 1011823 & intronic & & 0.674321 & 0.7045 \\
\hline & & rs7146 & SNP & $A / G$ & ontarget & 1014398 & exonic & $\begin{array}{l}\text { synonymous } \\
\text { SNV }\end{array}$ & 0.67512 & 0.6579 \\
\hline \multirow[t]{8}{*}{ POLL } & chr10 & rs1055364 & SNP & $\mathrm{C} / \mathrm{A}$ & ontarget & 103338730 & UTR3 & & 0.163339 & \\
\hline & & rs1055362 & SNP & $A / G$ & ontarget & 103338733 & UTR3 & & 0.163339 & \\
\hline & & rs3730477 & SNP & $\mathrm{G} / \mathrm{A}$ & ontarget & 103340056 & exonic & $\begin{array}{l}\text { nonsynonymous } \\
\text { SNV }\end{array}$ & 0.0998403 & 0.1678 \\
\hline & & rs3730476 & SNP & $A / G$ & ontarget & 103340144 & exonic & $\begin{array}{l}\text { synonymous } \\
\text { SNV }\end{array}$ & 0.163139 & 0.2306 \\
\hline & & rs3730475 & SNP & $A / G$ & ontarget & 103340179 & intronic & & 0.163339 & 0.232 \\
\hline & & rs3730474 & SNP & $\mathrm{T} / \mathrm{C}$ & flank150 & 103340235 & intronic & & 0.163139 & \\
\hline & & rs3730465 & SNP & $A / G$ & flank150 & 103343533 & intronic & & 0.164936 & \\
\hline & & rs3730462 & InDel & CTGTTG/C & ontarget & 103345941 & intronic & & 0.162939 & 0.2281 \\
\hline ASTN1 & chr1 & rs868002876 & InDel & $\mathrm{AT} / \mathrm{A}$ & ontarget & 176913216 & intronic & & 0.431909 & \\
\hline UGGT1 & chr2 & rs35069237 & InDel & $\mathrm{GT} / \mathrm{G}$ & ontarget & 128949841 & UTR3 & & 0.338858 & \\
\hline \multirow[t]{2}{*}{ NFATC1 } & chr18 & rs8096658 & SNP & $C / G$ & flank150 & 77156537 & intronic & & 0.328275 & \\
\hline & & rs56376587 & SNP & $\mathrm{A} / \mathrm{C}$ & flank150 & 77160235 & intronic & & 0.332268 & \\
\hline MTHFR & chr1 & rs11121832 & SNP & $\mathrm{T} / \mathrm{C}$ & flank150 & 11860120 & intronic & & 0.759185 & \\
\hline FCGR1B & chr1 & rs827371 & SNP & $\mathrm{T} / \mathrm{C}$ & flank150 & 120935661 & intronic & & & \\
\hline MAР3К8 & chr10 & rs3034 & SNP & $\mathrm{G} / \mathrm{A}$ & flank150 & 30749895 & UTR3 & & 0.882987 & \\
\hline \multirow[t]{2}{*}{ MGMT } & chr10 & rs2782888 & SNP & $T / G$ & flank150 & 131265328 & upstream & & 1 & \\
\hline & & rs55973415 & SNP & $\mathrm{G} / \mathrm{A}$ & flank150 & 131557750 & intronic & & 0.2143 & \\
\hline ZNF2929 & chr6 & rs563101504 & InDel & GACACAC/G & ontarget & 87925827 & intronic & & & \\
\hline
\end{tabular}




\begin{tabular}{|c|c|c|c|c|c|c|c|c|c|c|}
\hline Gene & $\mathrm{Chr}$ & SNP & Mut_type & Mutation & Location & Position & Func.ref & Exonicfunc.ref & $1 \mathrm{KG}$ & ExAC_AL \\
\hline & & rs9362415 & SNP & $A / G$ & ontarget & 87968565 & exonic & $\begin{array}{l}\text { nonsynonymous } \\
\text { SNV }\end{array}$ & 0.592851 & 0.5996 \\
\hline & & rs3734187 & SNP & $\mathrm{C} / \mathrm{T}$ & ontarget & 87969737 & exonic & $\begin{array}{l}\text { synonymous } \\
\text { SNV }\end{array}$ & 0.592652 & 0.5876 \\
\hline & & rs3812132 & SNP & $\mathrm{C} / \mathrm{G}$ & ontarget & 87969737 & exonic & $\begin{array}{l}\text { synonymous } \\
\text { SNV }\end{array}$ & 0.674121 & 0.5867 \\
\hline & & rs35541349 & InDel & G/GA & flank150 & 87969737 & UTR3 & & & \\
\hline FOS & chr14 & rs1063169 & SNP & $\mathrm{G} / \mathrm{T}$ & flank150 & 75747118 & intronic & & 0.113019 & \\
\hline ZNF346 & chr5 & rs11448853 & InDel & A/AG & flank150 & 176471286 & intronic & & 0.4742 & \\
\hline ERF & chr19 & rs61735151 & SNP & $\mathrm{G} / \mathrm{A}$ & ontarget & 42753283 & exonic & $\begin{array}{l}\text { synonymous } \\
\text { SNV }\end{array}$ & 0.0778754 & 0.0878 \\
\hline \multirow[t]{2}{*}{ ALKBH89 } & chr11 & rs589316 & SNP & $\mathrm{G} / \mathrm{A}$ & flank150 & 107402887 & intronic & & 0.35603 & \\
\hline & & rs71488261 & SNP & $\mathrm{T} / \mathrm{A}$ & flank150 & 107422440 & intronic & & 0.261981 & \\
\hline WDR55 & chr5 & rs2251860 & SNP & $\mathrm{T} / \mathrm{C}$ & ontarget & 140048209 & exonic & $\begin{array}{l}\text { synonymous } \\
\text { SNV }\end{array}$ & 0.508986 & 0.4722 \\
\hline AHSP & chr16 & rs10843 & SNP & $\mathrm{T} / \mathrm{C}$ & ontarget & 31540030 & UTR3 & & 0.0946486 & 0.1185 \\
\hline \multirow[t]{2}{*}{ DPCD } & chr10 & rs7911520 & SNP & $\mathrm{A} / \mathrm{G}$ & flank150 & 103354554 & intronic & & 0.169329 & \\
\hline & & rs7874 & SNP & $\mathrm{C} / \mathrm{T}$ & ontarget & 103361088 & exonic & $\begin{array}{l}\text { nonsynonymous } \\
\text { SNV }\end{array}$ & 0.0998403 & 0.168 \\
\hline
\end{tabular}

Table 3

Functional enrichment analysis of disease-causing DEGs using Metascape

\begin{tabular}{|c|c|c|c|c|}
\hline GO & Description $\log _{10} P$ Count Genes & & & \\
\hline C0002895 & Anemia Sickle cell & -5.9 & 6 & FOS|GYPA|MTHFR|SELP|AHSP|UGGT1 \\
\hline C0524702 & Pulmonary thromboembolisms & -5.5 & 3 & MTHFR|SELP|USP14 \\
\hline C0002875 & Cooley's anemia & -5.2 & 4 & GYPA|MTHFR|AHSP|UGGT1 \\
\hline C0272285 & Heparin-induced thrombocytopenia & -5.1 & 3 & FCGR1B|HNMT|SELP \\
\hline C0004135 & Ataxia telangiectasia & -4.8 & 5 & FOS|GYPA|MGMT|MTHFR|NFATC1 \\
\hline C0268138 & Xeroderma pigmentosum & -4.5 & 3 & MGMT|MTHFR|UGGT1 \\
\hline C0011615 & Dermatitis, Atopic & -4.5 & 6 & ASTN1|FOS|HNMT|MGMT|MTHFR|SELP \\
\hline C0008626 & Congenital-chromosomal disease & -4.5 & 6 & FCGR1B|FOS|MGMT|MTHFR|NFATC1|SELP \\
\hline C0278996 & Malignant chromosomal disease & -4.4 & 6 & FCGR1B|FOS|MGMT|MTHFR|NFATC1|SELP \\
\hline C3887461 & Head and neck carcinoma & -4.4 & 6 & FCGR1B|FOS|MGMT|MTHFR|NFATC1|SELP \\
\hline C0014170 & Endometrial neoplasms & -4.2 & 4 & MAP3K8|FOS|MGMT|MTHFR \\
\hline C0947751 & Vascular inflammations & -3.9 & 4 & SERPING1|MAP3K8|FOS|SELP \\
\hline C1704436 & Peripheral arterial diseases & -3.9 & 4 & MAP3K8|FOS|MTHFR|SELP \\
\hline C0011884 & Diabetic retinopathy & -3.7 & 5 & SERPING1|MAP3K8|FOS|SELP|MTHFR \\
\hline C0024814 & Marinesco-Sjogren syndrome & -3.7 & 3 & MAP3K8|MGMT|MTHFR \\
\hline С0333516 & Tumor necrosis & -3.7 & 4 & FOS|MGMT|MTHFR|SELP \\
\hline C3469521 & Fanconi anemia & -3.6 & 4 & GYPA|MGMT|MTHFR|SELP \\
\hline C4551686 & Malignant neoplasm of soft tissue & -3.6 & 5 & MAP3K8|FOS|MGMT|MTHFR|NFATC1 \\
\hline C0015625 & Fanconi anemia & -3.5 & 4 & GYPA|MGMT|MTHFR|SELP \\
\hline C0398623 & Thrombophilia & -3.5 & 3 & SERPING1|MTHFR|SELP \\
\hline
\end{tabular}


The Thr105lle (rs11558538) polymorphism in the HNMT gene (ch2: $138759649 \mathrm{C}>\mathrm{T}$, rs11558538, adjusted P-value $=1.2 \times 10^{-9}$ ) was the biggest difference identified in a gene, and should result in nonsense-mediated decay and loss function of this protein. The 3D location is shown in Fig. 5A and Fig. 5B. The variant was positioned in the a-helix, where its side chain hydroxyl formed two hydrogen bonds with a backbone oxygen after mutation, causing a marked decrease in the levels of both HNMT enzymatic activity and immunoreactive protein[26, 27]. HNMT is an enzyme that has been implicated in neurotransmission by inactivating histamine in the central nervous system[28]. However, histamine increases vascular permeability through the histamine $\mathrm{H} 1$ receptor to activate nerve endings, relaxing vascular smooth muscle[29].

\subsection{Molecular Docking}

The drug-target interactions for HNMT were predicted using DGIdb, and the results are presented in Table 4, providing a theoretical therapeutic mechanism for VTE prevention. Six drugs targeting HNMT have been predicted for VTE, including Amodiaquine, Harmaline, Aspirin, Metoprine, Dabigatran, and Diphenhydramine.

Molecular docking analysis was attempted to assess the potential noncovalent binding of HNMT with these small molecules drugs. In general, a lower binding energy indicated a stronger binding between HNMT and a compound. Table 4 shows the six drugs that best interfaced with HNMT. To visualize these docking results, the 3D interaction diagrams of HNMT and their corresponding best-matched drugs were drawn, as shown in Fig. 6 . These drugs, such as Aspirin and Dabigatran, have been utilized to recanalize vessels and prevent thrombi growth clinically in VTE patients[30-32]. The 3D interaction diagram of Aspirin at the active site of HNMT revealed that this interaction was stable through forming hydrogen bonds with the key residues Lys55 and Lys135 (Fig. $6 \mathrm{~B}$ ). Aspirin is commonly administered to inhibit platelet aggregation and prevent thrombus formation[33]. Additionally, three hydrogen bonds formed with residues Phe9, Tyr15, and Ser91 contributed to stabilizing the interaction between Dabigatran and HNMT. Dabigatran has been approved for use in orthopedic surgery, venous thromboprophylaxis, acute VTE treatment, and extended prevention of recurrent VTE[34]. Our data had shown that HNMT can potentially become a new target for VTE treatment.

Table 4

Candidate drugs targeted HNMT

\begin{tabular}{|llllll|}
\hline Gene & Drug & Sources & PMIDs & Binding Energy (kcal. mol-) & Binding Residues \\
\hline HNMT & Amodiaquine & DrugBank; & 6789797 & -2.48 & GLN197; \\
& & & & ASP203 \\
HNMT & Aspirin & DrugBank & 19178400 & -3.24 & LYS55; \\
& & & & LYS135 \\
HNMT & Harmaline & PharmGKB & 1530666 & -5.07 & GLU28 \\
HNMT & Metoprine & TTD;DTC & 10592235 & -3.65 & GLN192; \\
HNMT & Dabigatran & & & & ASP194 \\
HNMT & Diphenhydramine & DrugBank & 23896426 & -4.69 & PHE9;TYR15;SER91 \\
\hline
\end{tabular}

\section{Discussion}

In the present study, transcriptomics and proteomics technology were used to explore the potential pathways and pathogenic mutations of VTE occurrence. High-throughput pharmacology and molecular docking may allow for the investigation of novel biomarkers for detecting this complex diseases[35].

We first studied VTE by downloading transcriptome-wide expression data from the GEO database and a total of 232 DEGs were identified. Results of GO analysis of the gene enrichment in these datasets showed that the VTE-associated DEGs were significantly enriched in RNA catabolic process. Wang, HX found previously that RPL9, RPL35, and RPS7 were hub genes in the PPI network of GSE13985, which was used to identify potential markers of atherosclerosis development[36]. Interestingly, a study from Mi, YH reported that the major risk factors for atherothrombotic disease were also significantly associated with VTE, which contributes to the explanation of why atherosclerosis is an independent risk factor for VTE[37]. KEGG pathway analysis revealed that the DEGs related to VTE were mainly enriched in the ribosome pathway. Recent evidence has suggested that the ribosome affects the translation of platelets, platelet aggregation, and resultant thrombus formation[38, 39]. It may be reasonable for us to then hypothesize that ribosomal proteins might have crucial functions in VTE development. However, there is no direct evidence that RNA catabolic processes and the ribosome pathway are directly involved in VTE.

To verify the above results, whole-exome sequencing (WES) was performed to detect the pathogenic mutations of DEGs. POLL encodes the novel DNA polymerase lambda and the mutation of POLL (rs3730477) encoded R438W Pol $\lambda$ leading to genomic instability and mutagenesis in cells[40]. DPCD (rs7874) is named from an uncharacterized genomic region surrounding POLL. DPCD is a novel gene in primary ciliary dyskinesia and severe cases can induce pulmonary embolism[41]. ZNF292 (rs9362415) acts as a transcription factor and plays an important role in DNA recognition and apoptosis regulation[42]. However, little is known about the role of DNA related functions in VTE. Notably, we discovered that HNMT (rs11558538) polymorphism was the greatest differentially expressed factor in this study. As is well-known, HNMT is implicated in neurotransmission by inactivating histamine, and histamine has been argued to relax vascular smooth muscle[29]. From a protein structure analysis, we found that the Thr105lle mutation results in hydrogen bonds in the structure 
of HNMT being disrupted, resulting in loss-of-function mutations[43]. The 3D structure diagram of HNMT showed quite different in protein conformations between the Thr105 and lle105 variants. Furthermore, a list of drugs targeting HNMT with potential therapeutic efficacy against VTE were selected, most notably Aspirin and Dabigatran. As a consequence, we inferred that the high expression of mutated HNMT acted on vascular smooth muscle and may further promote vasoconstriction and thrombosis through RNA catabolic process and the ribosome. However, the mechanisms of these genes and drugs in VTE are still unclear. In future work, we hope to verify our conclusions experimentally to elucidate the effects of Thr105lle (rs11558538) in HNMT for VTE.

\section{Conclusions}

The current study was designed to investigate potential DEGs and genetic variant of DEGs in VTE (Fig. 7). RNA catabolic process and ribosomes pathway identified through integrated bioinformatic analysis of GSE48000 and GSE19151 datasets may play crucial roles in the development of VTE. Additionally, Thr105lle (rs11558538) polymorphism in HNMT was identified as a risk factor for VTE in the mechanism of damage and dysfunctional to the vascular endothelial cell and vascular smooth muscle. In the future, more in-depth investigation about the mechanism of these candidate genes is warranted for VTE.

\section{Abbreviations}

VTE: venous thromboembolism; DEGs: Differentially expressed genes; GEO: Gene Expression Omnibus; DEGs: Differentially expressed genes; WES: Wholeexome Sequencing; KEGG: Kyoto Encyclopedia of Genes and Genomes; WES: whole-exome sequencing; HNMT: Histamine N-Methyltransferase; PCA: Principal component analysis; BPs: Biological Processes; MFs: Molecular Functions; CCs: cellular components; SNPs: Single-nucleotide Polymorphisms; INDELs: insertion-deletions.

\section{Declarations}

Acknowledgments

We thank the many who participated in this VTE study and funded this work.

Authors' contributions

JD were responsible for acquisition of data, analysis and interpretation of data. $Y Z$ and $Y C$ critically revised the work. YC were responsible for the conception and design of the study and final approval of the version to be submitted. The manuscript was written by JD. All authors read and approved the final manuscript.

Funding

This project was supported by grants from the National Natural Science Foundation of China (Award No:81472990), the Clinical Research Project of Shanghai Municipal Commission of Health and Family Planning(Award No:201840095) and the Clinical Research Project of Shanghai Municipal Commission of Health and Family Planning((Award No:20214Y0332).

Availability of data and materials

Not applicable.

Ethics approval and consent to participate

All patients participating in this study were informed, signed informed consents and voluntarily participated, and this study was approved by the ethics committee of the Shanghai Institute of Planned Parenthood Research and ethical committees of participating hospitals.

Consent for publication

Not applicable.

Competing interests

All authors declare that they have no competing interests.

Author details

NHC Key Lab of Reproduction Regulation (Shanghai Institute for Biomedical and Pharmaceutical Technologies), Fudan University, Shanghai, 200032, China.

\section{References}

1. Wang W, Zhu X, Du X, Xu A, Yuan X, Zhan Y, et al. MiR-150 promotes angiogensis and proliferation of endothelial progenitor cells in deep venous thrombosis by targeting SRCIN1. Microvasc Res. 2019;123:35-41.

2. Evangelista MS, Slompo K, Timi JRR. Venous Thromboembolism and Route of Delivery - Review of the Literature. Rev Bras Ginecol Obstet. 2018;40(3):156-62.

3. Xu Y, Siegal DM, Anand SS. Ethnoracial variations in venous thrombosis: Implications for management, and a call to action. J Thromb Haemost. 2020. 
4. Lee LH, Gallus A, Jindal R, Wang C, Wu CC. Incidence of Venous Thromboembolism in Asian Populations: A Systematic Review. Thromb Haemost. 2017; 117(12):2243-60.

5. Riedl J, Ay C. Venous Thromboembolism in Brain Tumors: Risk Factors, Molecular Mechanisms, and Clinical Challenges. Semin Thromb Hemost. 2019;45(4):334-41.

6. Moser G, Guettler J, Forstner D, Gauster M. Maternal Platelets-Friend or Foe of the Human Placenta? Int J Mol Sci. 2019;20(22).

7. Zöller B, Svensson PJ, Dahlbäck B, Lind-Hallden C, Hallden C, Elf J. Genetic risk factors for venous thromboembolism. Expert Rev Hematol. 2020;13(9):971-81.

8. Kumar DR, Hanlin E, Glurich I, Mazza JJ, Yale SH. Virchow's contribution to the understanding of thrombosis and cellular biology. Clin Med Res. 2010;8(34):168-72.

9. Rouhi-Broujeni H, Pourgheysari B, Hasheminia AM. Association of Homozygous Thrombophilia Polymorphisms and Venous Thromboembolism in Shahrekord, Iran. Tanaffos. 2016;15(4):218-24.

10. Lindström S, Brody JA, Turman C, Germain M, Bartz TM, Smith EN, et al. A large-scale exome array analysis of venous thromboembolism. Genet Epidemiol. 2019;43(4):449-57.

11. Almohammed OA, Lai L, Khanfar NM, Bleidt B, Aljadhey H. Trends of cancer-associated venous thromboembolism (VTE) in the United States (20052014). Thromb Res. 2019;182:110-5.

12. Clough E, Barrett T. The Gene Expression Omnibus Database. Methods Mol Biol. 2016;1418.

13. Lewis DA, Suchindran S, Beckman MG, Hooper WC, Grant AM, Heit JA, et al. Whole blood gene expression profiles distinguish clinical phenotypes of venous thromboembolism. Thromb Res. 2015;135(4):659-65.

14. Lewis DA, Stashenko GJ, Akay OM, Price LI, Owzar K, Ginsburg GS, et al. Whole blood gene expression analyses in patients with single versus recurrent venous thromboembolism. Thromb Res. 2011;128(6):536-40.

15. Gautier L, Cope L, Bolstad BM, Irizarry RA. affy-analysis of Affymetrix GeneChip data at the probe level. Bioinformatics. 2004;20(3):307-15.

16. Lun ATL, Perry M, Ing-Simmons E. Infrastructure for genomic interactions: Bioconductor classes for Hi-C, ChIA-PET and related experiments. F1000Res. 2016;5:950.

17. Jolliffe IT, Cadima J. Principal component analysis: a review and recent developments. Philos Trans A Math Phys Eng Sci. 2016;374(2065):20150202.

18. Ritchie ME, Phipson B, Wu D, Hu Y, Law CW, Shi W, et al. limma powers differential expression analyses for RNA-sequencing and microarray studies. Nucleic Acids Res. 2015;43(7):e47.

19. Kumar N, Hoque MA, Sugimoto M. Robust volcano plot: identification of differential metabolites in the presence of outliers. BMC Bioinformatics. 2018;19(1):128.

20. Zhao Y, Fu G, Wang J, Guo M, Yu G. Gene function prediction based on Gene Ontology Hierarchy Preserving Hashing. Genomics. 2019;111(3):334-42.

21. Kanehisa M, Sato Y, Kawashima M, Furumichi M, Tanabe M. KEGG as a reference resource for gene and protein annotation. Nucleic Acids Res. 2016;44(D1):D457-D62.

22. Yu G, Wang L-G, Han Y, He Q-Y. clusterProfiler: an R package for comparing biological themes among gene clusters. OMICS. 2012;16(5):284-7.

23. Zhou Y, Zhou B, Pache L, Chang M, Khodabakhshi AH, Tanaseichuk O, et al. Metascape provides a biologist-oriented resource for the analysis of systemslevel datasets. Nat Commun. 2019;10(1):1523.

24. Cotto KC, Wagner AH, Feng YY, Kiwala S, Coffman AC, Spies G, et al. DGIdb 3.0: a redesign and expansion of the drug-gene interaction database. Nucleic Acids Res. 2018;46(D1):D1068-d73.

25. Morris GM, Huey R, Lindstrom W, Sanner MF, Belew RK, Goodsell DS, et al. AutoDock4 and AutoDockTools4: Automated docking with selective receptor flexibility. J Comput Chem. 2009;30(16):2785-91.

26. Chen Y, Cao B, Ou R, Wei Q, Chen X, Zhao B, et al. Determining the Effect of the HNMT, STK39, and NMD3 Polymorphisms on the Incidence of Parkinson's Disease, Amyotrophic Lateral Sclerosis, and Multiple System Atrophy in Chinese Populations. J Mol Neurosci. 2018;64(4):574-80.

27. Jiménez-Jiménez FJ, García-Martín E, Alonso-Navarro H, Martínez C, Zurdo M, Turpín-Fenoll L, et al. Thr105lle (rs11558538) polymorphism in the histamine-1-methyl-transferase (HNMT) gene and risk for restless legs syndrome. J Neural Transm (Vienna). 2017;124(3):285-91.

28. Meza-Velázquez R, López-Márquez F, Espinosa-Padilla S, Rivera-Guillen M, Ávila-Hernández J, Rosales-González M. Association of diamine oxidase and histamine $\mathrm{N}$-methyltransferase polymorphisms with presence of migraine in a group of Mexican mothers of children with allergies. Neurologia. 2017;32(8):500-7.

29. Huang M, Wang X, Xing B, Yang H, Sa Z, Zhang D, et al. Critical roles of TRPV2 channels, histamine H1 and adenosine A1 receptors in the initiation of acupoint signals for acupuncture analgesia. Sci Rep. 2018;8(1):6523-.

30. Hanafy AS, Abd-Elsalam S, Dawoud MM. Randomized controlled trial of rivaroxaban versus warfarin in the management of acute non-neoplastic portal vein thrombosis. Vascul Pharmacol. 2019;113:86-91.

31. Tan ZK, Casipit P, Pandit NS. PO-10 - Lung cancer with tumour thrombosis: a poor prognosticator. Thromb Res. 2016;140(Suppl 1):180.

32. Suzuki K, Yanagimura T, Ohashi K, Kagamu H, Takada T. Bevacizumab-induced Aortic Arterial Thrombosis. Intern Med. 2018;57(20):2987-90.

33. Kang G, Zhang H. Aspirin for Postoperative Venous Thromboembolism Prophylaxis. J Gen Intern Med. 2020;35(9):2778.

34. Schulman S, Kearon C, Kakkar AK, Mismetti P, Schellong S, Eriksson H, et al. Dabigatran versus warfarin in the treatment of acute venous thromboembolism. N Engl J Med. 2009;361(24):2342-52.

35. Azad RK, Shulaev V. Metabolomics technology and bioinformatics for precision medicine. Brief Bioinform. 2019;20(6):1957-71. 
36. Wang HX, Zhao YX. Prediction of genetic risk factors of atherosclerosis using various bioinformatic tools. Genet Mol Res. 2016;15(2).

37. Mi Y, Yan S, Lu Y, Liang Y, Li C. Venous thromboembolism has the same risk factors as atherosclerosis: A PRISMA-compliant systemic review and metaanalysis. Medicine. 2016;95(32):e4495-e.

38. Rowley JW, Weyrich AS. Ribosomes in platelets protect the messenger. Blood. 2017;129(17):2343-5.

39. Wang H, Song N-P, Li J-P, Wang Z-H, Ti Y, Li Y-H, et al. The microvesicle/CD36 complex triggers a prothrombotic phenotype in patients with non-valvular atrial fibrillation. J Cell Mol Med. 2020;24(13):7331-40.

40. Nemec AA, Bush KB, Towle-Weicksel JB, Taylor BF, Schulz V, Weidhaas JB, et al. Estrogen Drives Cellular Transformation and Mutagenesis in Cells Expressing the Breast Cancer-Associated R438W DNA Polymerase Lambda Protein. Mol Cancer Res. 2016;14(11):1068-77.

41. Nandhakumar A, Silverman GL. Acute hypoxemia in a parturient with primary ciliary dyskinesia following the administration of intravenous oxytocin: a case report. Can J Anaesth. 2013;60(12):1218-21.

42. Mirzaa GM, Chong JX, Piton A, Popp B, Foss K, Guo H, et al. De novo and inherited variants in ZNF292 underlie a neurodevelopmental disorder with features of autism spectrum disorder. Genet Med. 2020;22(3):538-46.

43. Verhoeven WMA, Egger JIM, Janssen PKC, van Haeringen A. Adult male patient with severe intellectual disability caused by a homozygous mutation in the HNMT gene. BMJ Case Rep. 2020;13(12).

\section{Figures}

A

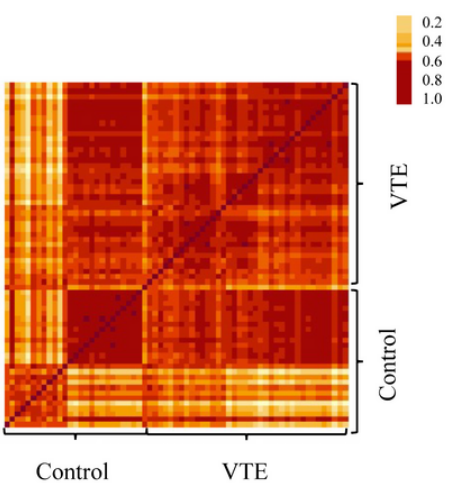

$\mathrm{C}$

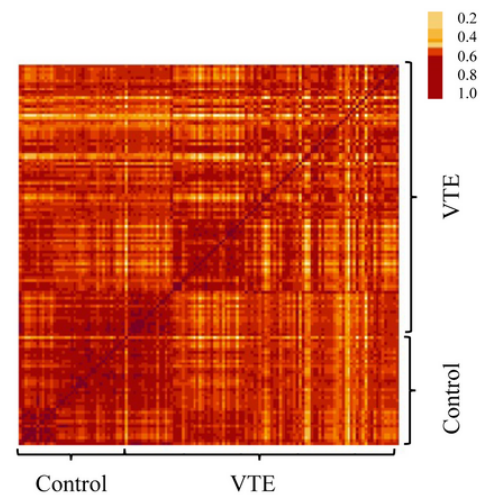

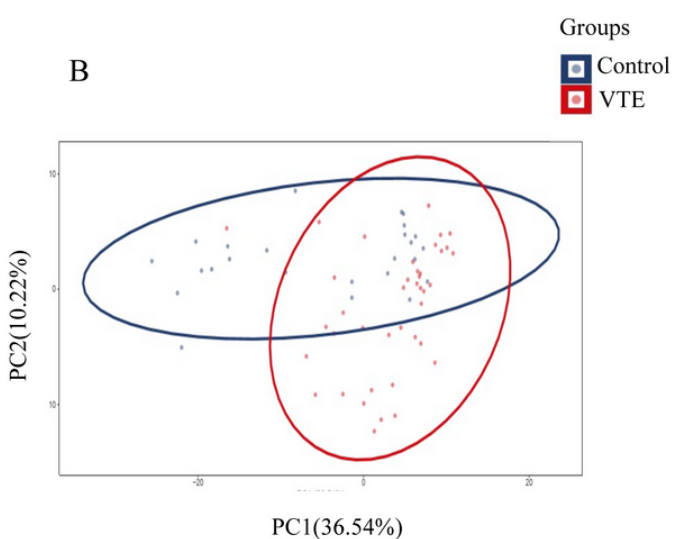

Groups
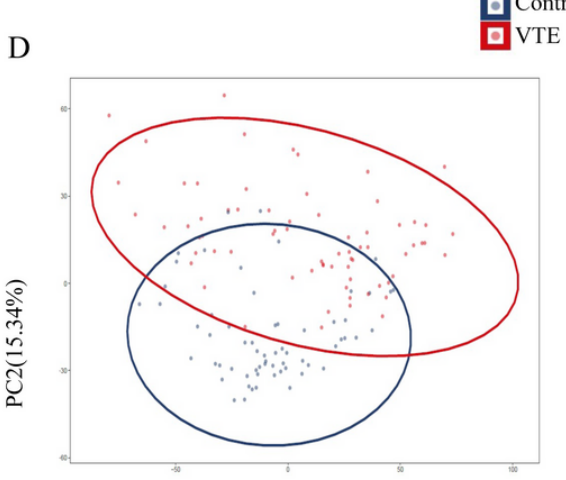

$\operatorname{PC} 1(32.72 \%)$

\section{Figure 1}

Pearson's correlation test and PCA on GSE48000 and GSE19151 data. (A) Pearson's correlation test for GSE48000. The color reflects the intensity of the correlation. (B) PCA of samples from the GSE48000 dataset. Principal component 1 values for VTE samples are plotted on the $X$ axis, and principal component 2 values for control samples are plotted on the $Y$ axis. The closer the distance between the two groups, the smaller the differences between the two groups. (C) Pearson's correlation test for GSE19151. The color reflects the intensity of the correlation. (D) PCA of samples from the GSE19151 dataset. 
A

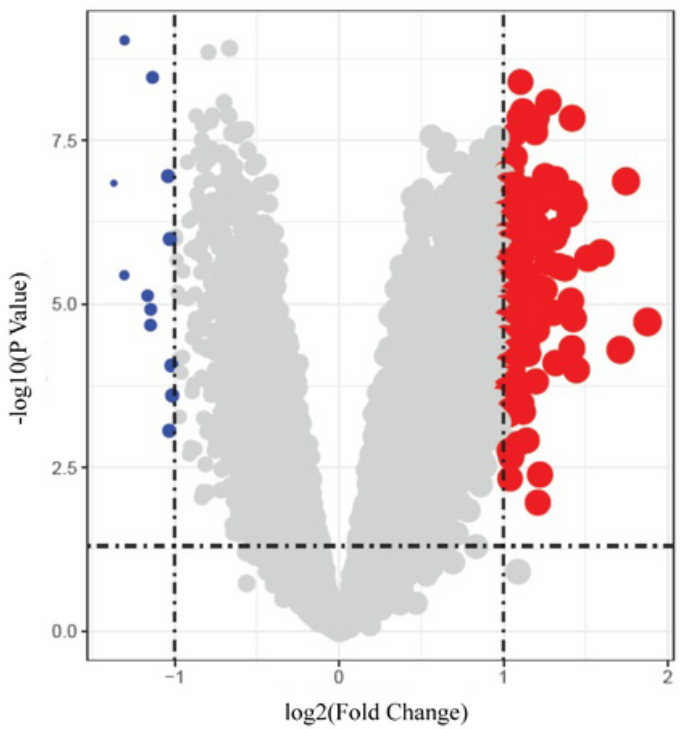

B

Regulated

- Down

- No significant

- Up
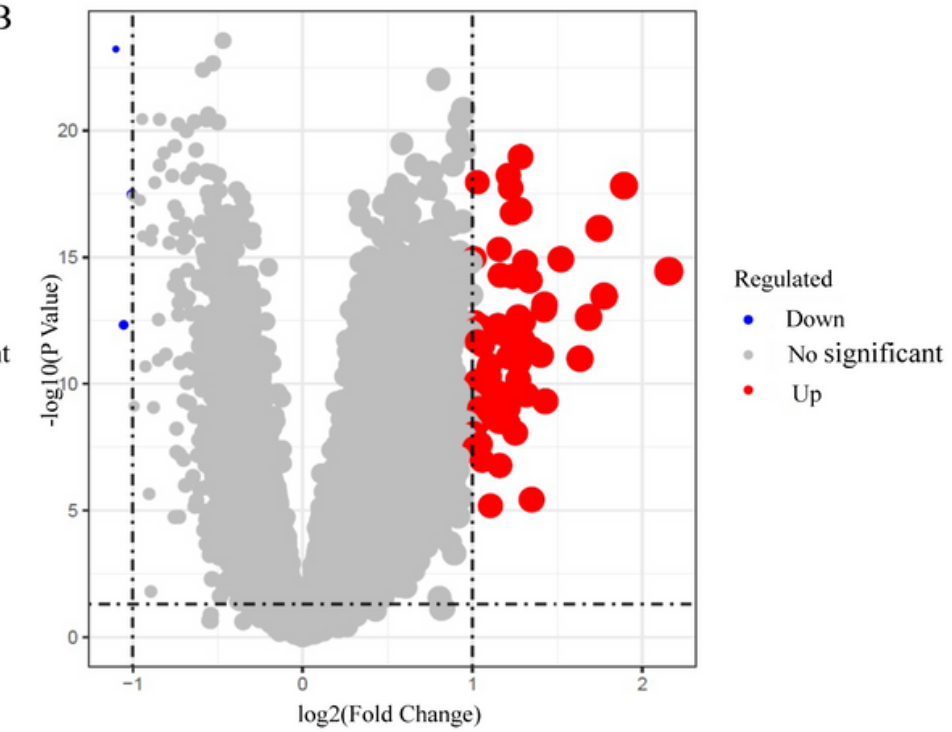

Figure 2

Volcano plot of VTE DEGs. Red, upregulated DEGs with log2FC $>1$ and adjusted P-value $<0.05$. Green, downregulated DEGs with log2FC $<-1$ and adjusted Pvalue $<0.05$. (A) Volcano plot illustrating the DEGs of the GSE48000 dataset. (B) Volcano plot illustrating the DEGs of the GSE19151 dataset.

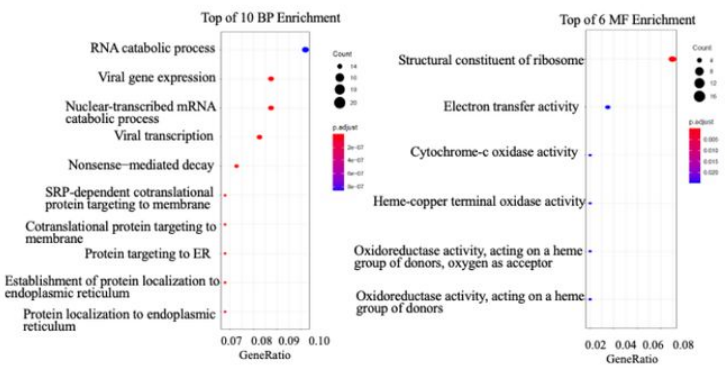

$\mathrm{C}$

D
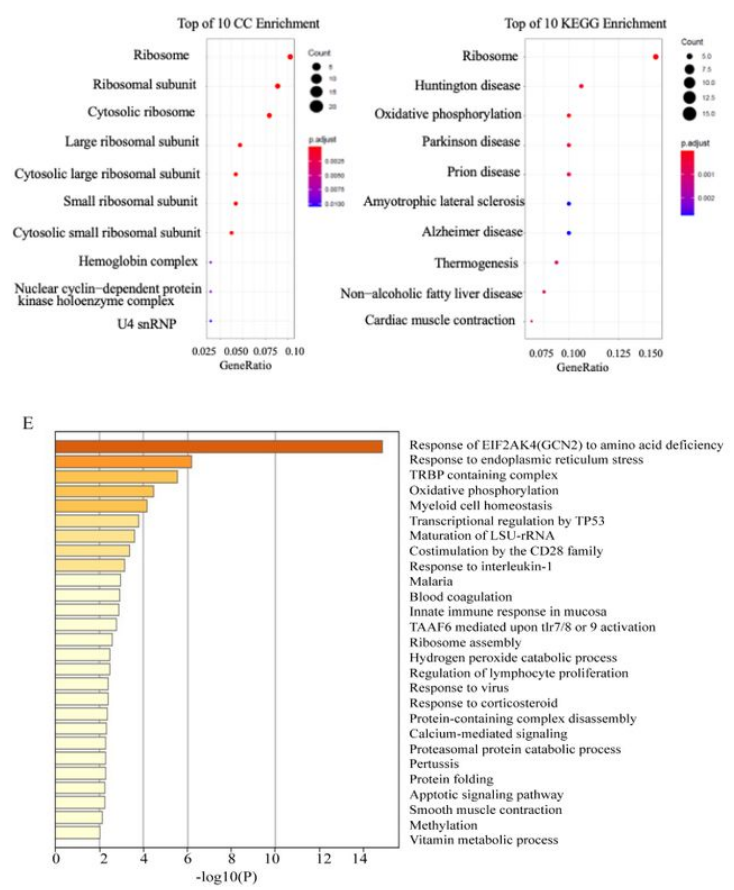

Figure 3 
Bubble map for GO and KEGG pathway analyses of DEGs. P-value $<0.05$ was considered statistically significant. (A) Biological processes, (B) molecular function, (C) cell component, (D) KEGG pathways, (E) heatmap of enriched terms across DEGs, colored by P-values, via Metascape.

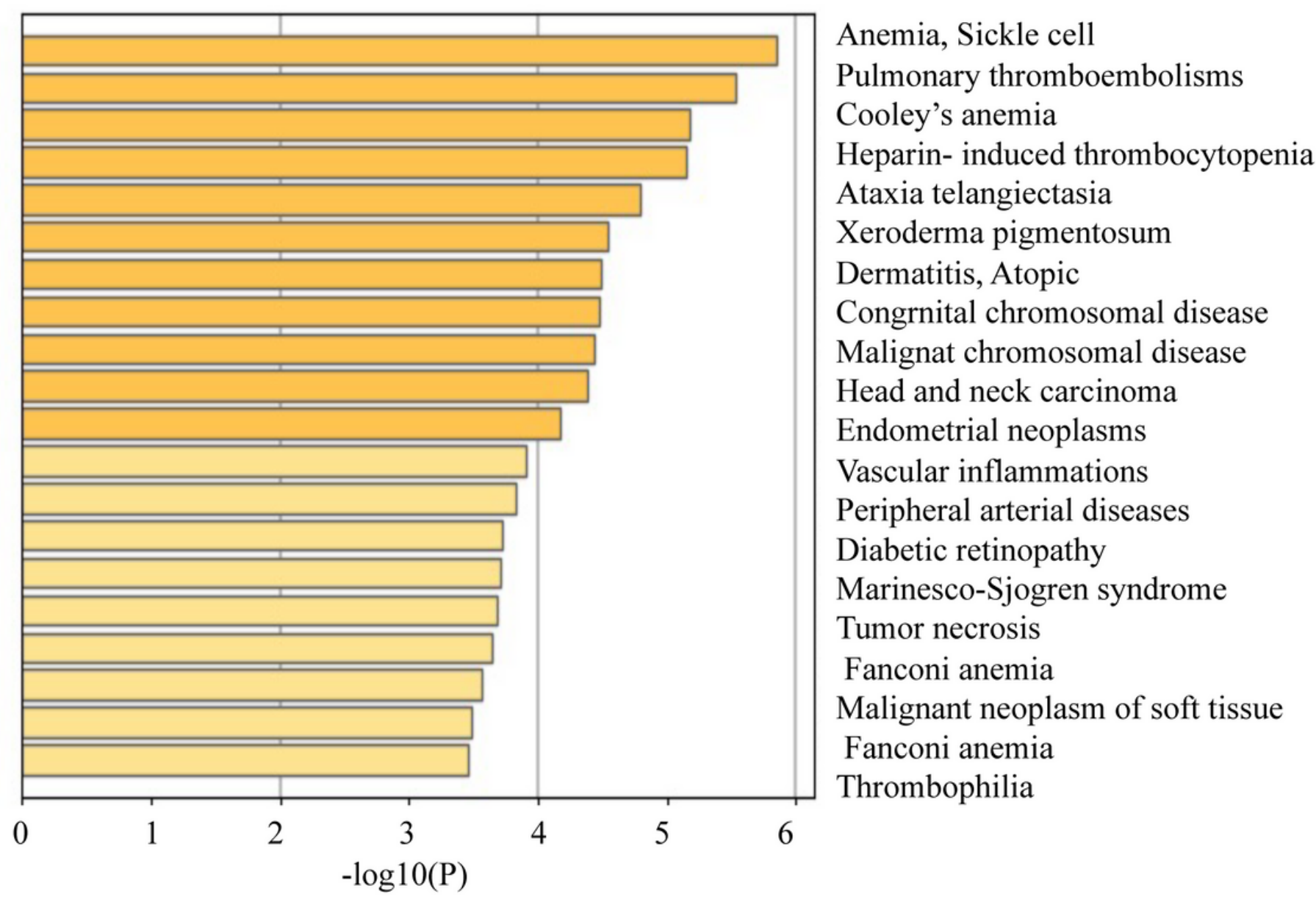

Figure 4

Heatmap of enriched terms across disease-causing DEGs, via Metascape. 

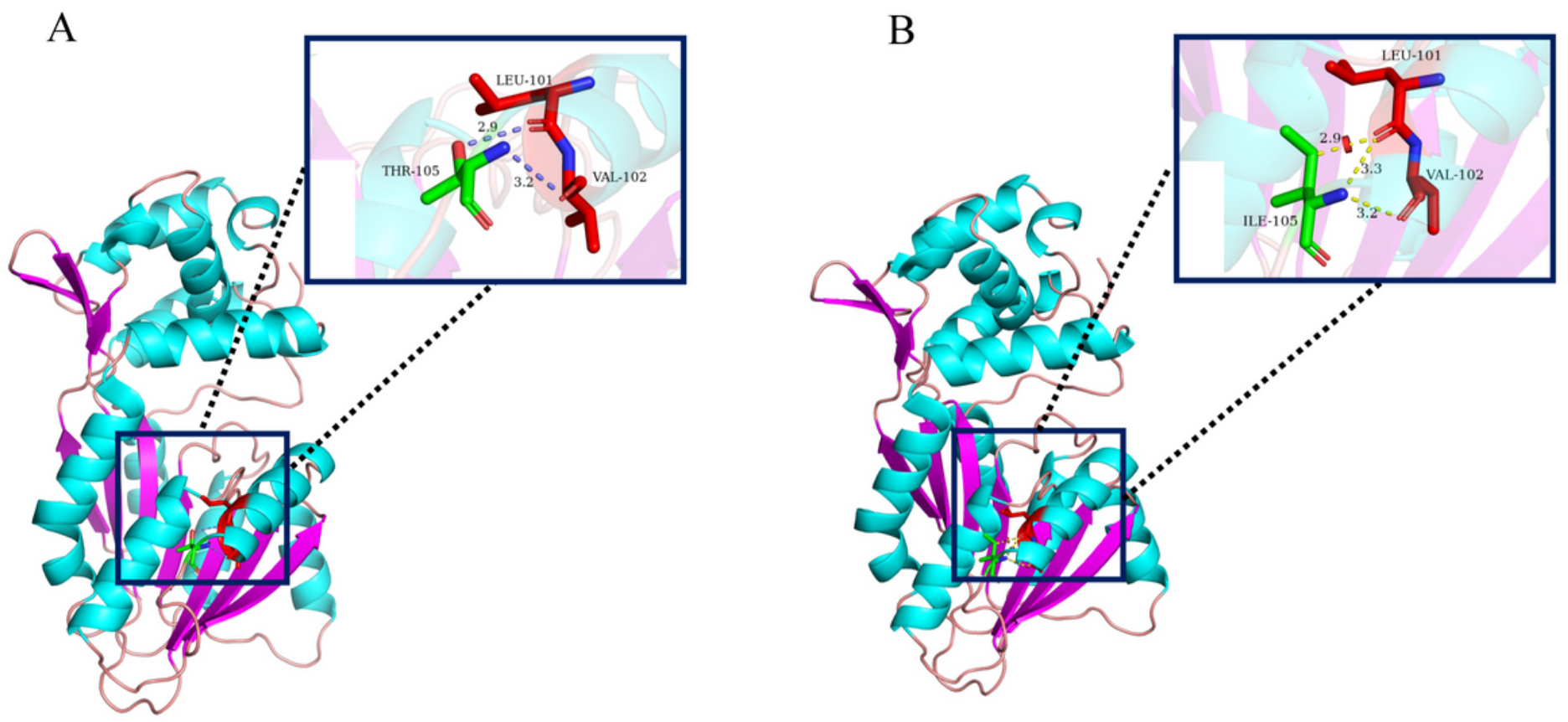

Figure 5

3D structure of HNMT. (A) Diagram of the HNMT structure depicting the location of Thr105. (B) Diagram of the HNMT structure depicting the location of the lle105 mutation.
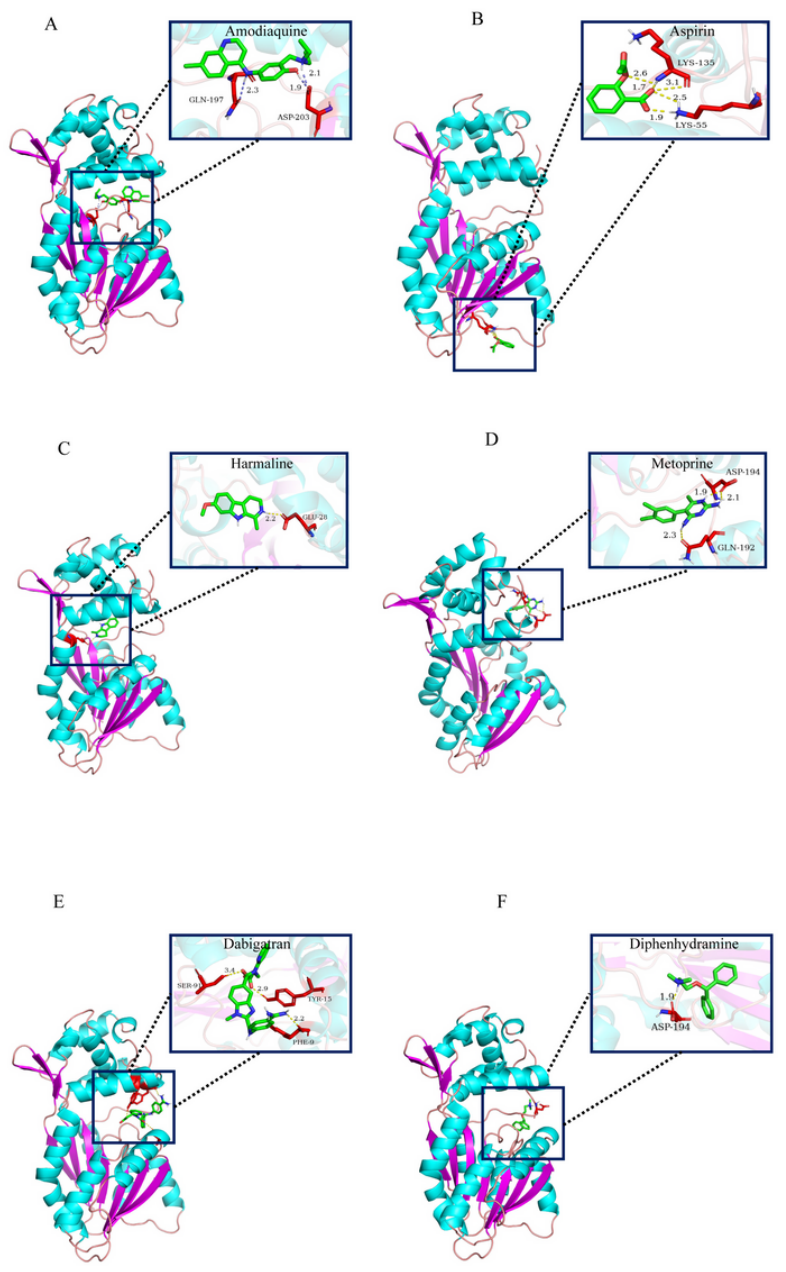
Figure 6

The 3D structure diagram of the drugs and HNMT with the active sites. (A) Structures of the pocket of binding between HNMT and Amodique. (B) Structures of the binding between HNMT and Aspirin. (C) Structures of the binding between HNMT and Harmaline. (D) Structures of the binding between HNMT and Metropine. (E) Structures of the binding between HNMT and Dabigatran. (F) Structures of the binding between HNMT and Diphenhydramine.

\section{HNMT mutation (THR105ILE) polymorphism in VTE}

\section{Wild type}

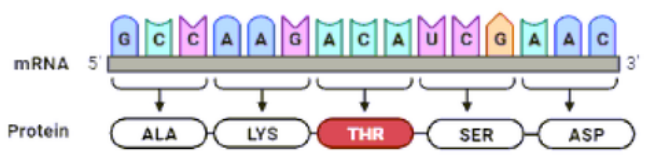

Missense mutation

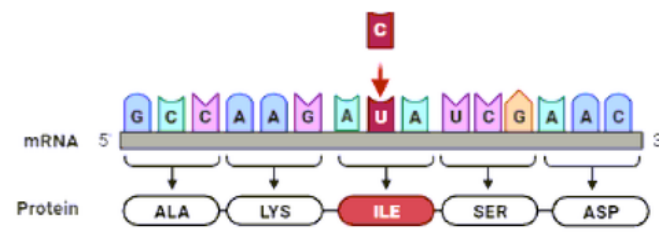

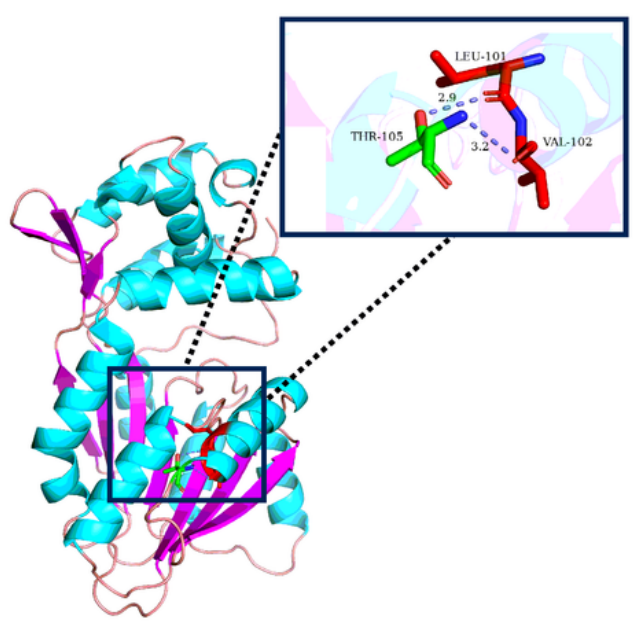

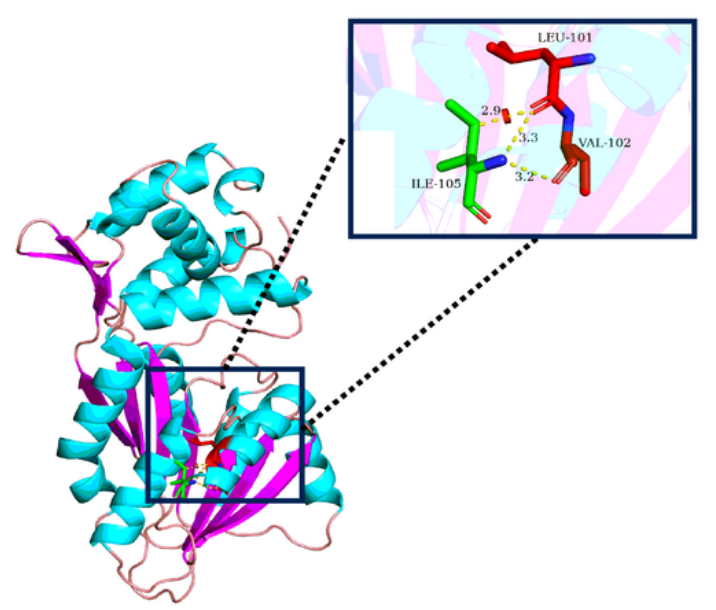

Figure 7

Summary diagram regarding the genetic variant of DEGs in VTE.

\section{Supplementary Files}

This is a list of supplementary files associated with this preprint. Click to download.

- GraphicalAbstract.tif

- Supplementarylnformation.docx 